1 Hacettepe Journal of Mathematics and Statistics

h Volume 47 (3) (2018), 625-635

\title{
Exponential decay for a neutral one-dimensional viscoelastic equation
}

\author{
Nasser-eddine Tatar *
}

\begin{abstract}
In this work we consider a viscoelastic string subject to a delay of neutral type. The delay occurs in the second time derivative. Although delays are known by their destructive nature, here we prove an exponential decay result. We shall use the multiplier method and modify the classical energy by judicious choices of other functionals. This would lead to an appropriate differential inequality which allows us to conclude. It seems that this issue has never been discussed before in the literature.
\end{abstract}

Keywords: Exponential decay, modified energy, multiplier technique, neutral delay, stability.

2000 AMS Classification: 35L05, 34K40

Received: 07.03.2017 Accepted : 19.04.2017 Doi : 10.15672/HJMS.2017.479

\section{Introduction}

Time-delay systems arouse the curiosity of many researchers in the last three decades. They appear often in real-world engineering problems and population dynamics $[14,15,17$, 36]. Loosely speaking we have a delay whenever the response to an applied force is involved. Models with time-lags in their variables are often referred to as 'Functional Differential Equations' (FDEs) [14,15,17,36]. A special subclass of FDEs is the class of 'Neutral Delay Differential Equations' (NDDEs) [9,14,15,17,18,35,36,40]. It is composed of those differential equations involving derivatives (possibly the highest one) evaluated at previous time.

Researchers have been studying such FDEs not only because they constitute a natural extension of related ordinary differential equations but also and mainly because of their appearance in many real-world problems: heat exchange, ecology, electrodynamics, neutral networks, etc [14,15,17,36-38].

${ }^{*}$ King Fahd University of Petroleum and Minerals, Department of Mathematics and Statistics, Dhahran 31261, Saudi Arabia

Email : tatarn@kfupm.edu.sa 
It has been shown that time-delay may trigger complex behaviors of instability. Even small delays can destabilize a system which was initially stable $[2,6-9,12,16]$. This phenomenon provokes oscillation, imperfection and discomfort leading sometimes to destructions. To solve this problem, researchers have been trying to stabilize the system by different ways. Although many controls have been devised so far and many methods and results have been found, NDDEs remains a class of differential equations which is not well understood. It is also worth mentioning that besides the fact that systems are very reactive to small delays, on the contrary, they can be stabilized by 'large' neutral delays. In fact, neutral delays are sometimes deliberately inserted into the systems to improve the performance of the structure.

Of concern here is the problem

$$
\left\{\begin{array}{l}
u_{t t}(t, x)-p \int_{0}^{x} u_{t t}(t-\tau, y) d y=u_{x x}(t, x)-\int_{0}^{t} g(t-s) u_{x x}(s, x) d s \\
\text { in }(0, \infty) \times(0,1) \\
u(t, x)=0, t \in(0, \infty), x=0,1 \\
u(0, x)=u_{0}(x), u_{t}(0, x)=u_{1}(x), x \in(0,1),
\end{array}\right.
$$

where the delay in the first integral in the equation is of neutral type and the second integral is a viscoelastic term (or a memory term). The (relaxation) function $g$ is a (nonincreasing) continuously differentiable function and $p$ is a real number. The functions $u_{0}(x)$ and $u_{1}(x)$ are given initial data and $\tau>0$.

Second order NDDE appear, in general, in the study of vibrating masses attached to an elastic bar and also (as the Euler equation) in some variational problems [14,15,17,37].

Searching in the literature we were unable to find studies on the asymptotic behavior or stability of this problem. The ones we have found are concerned with either ordinary differential equations (see $[14,15,18,33-35]$ and the references therein), or first order partial differential equations $[26,32]$. The few treated second order problems are concerned with delays in the state function or its first derivative rather than in the second derivative.

The equation in (1.1) without the neutral delay (i.e. $p=0$ ) ia a one-dimensional viscoelastic equation based on the Boltzmann principle [1]. Under certain assumptions, Boltzmann derived the constitutive relationship between the stress and the strain in the form

$$
\sigma(t, x)=a \varepsilon(t, x)+\int_{-\infty}^{t} g(t-s)[\varepsilon(t, x)-\varepsilon(s, x)] d s .
$$

This relation describes adequately viscoelastic materials such as polymers, certain glasses, rubber, concrete ans soils. In case the kernel is integrable then this relation becomes

$$
\sigma(t, x)=b \varepsilon(t, x)-\int_{-\infty}^{t} g(t-s) \varepsilon(s, x) d s, b=a+\int_{0}^{\infty} g(s) d s .
$$

Notice that the Newtonian viscosity may result as a limiting case or simply by taking $g=-\delta^{\prime}$ where $\delta$ is the Dirac delta function.

The nonlinear case where $u_{x x}$ is replaced by $\varphi\left(u_{x}\right)_{x}$ and $\psi\left(u_{x}\right)_{x}$ in the first and second terms in the right hand side of (1.1) has been investgated by Coleman and Gurtin [4]. The authors discussed the competition between the memory effect and the nonlinear elastic response. Subsequently, several other researchers worked on this issue (see Christensen [3]). We also mention C. M. Dafermos, J. A. Nohel, W. J. Hrusa, H. Engler, M. Renardy, V. J. Mizel W. A. Day and A. C. Pipkin to cite but a few (see $[5,8,19,24]$ ). They have contributed considerably in developing this theory. Then, P. Cannarsa, D. Sforza, M. Fabrizio, B. Lazzari, C. Giorgi, J. E. Munoz Rivera, M. M. Cavalcanti, V. N. Domingos Cavalcanti, P. Martinez, V. Pata and many others took over the study of the (linear) problem. Some of them are cited in [13, 2-23, 27-31]. Roughly and briefly, it has been shown that the memory term produces an extremely weak dissipation but capable of 
stabilizing the system. The rate of decay of the (classical) energy is shown to be of the same type as the one of the kernel but does not exceed it. The memory term may also take it over nonlinear sources and therefore prevent blow up in finite time.

In the next section, we shall introduce the functionals we will work with. Section 3 is devoted to the statement and proof of our result.

\section{Preliminaries}

In this section we prepare some material which will be used to prove our result. The existence and uniqueness for this type of problems and even more general ones has been discussed in $[10,11,39,40]$. Classical solutions exist in

$$
D(A):=\left\{u \in H^{2}(0,1): u(0)=u(1)=0\right\}
$$

where $A w:=w^{\prime \prime}$, for regular initial data.

Let us define the functionals

$$
\begin{aligned}
\mathcal{E}(t) & :=\frac{1}{2}\left\{\left\|u_{t}(t)-p \int_{0}^{x} u_{t}(t-\tau, y) d y\right\|^{2}\right. \\
+(1 & \left.\left.-\int_{0}^{t} g(s) d s\right)\left\|u_{x}\right\|^{2}+\int_{0}^{1}\left(g \square u_{x}\right) d x\right\} \\
\phi_{1}(t) & :=-\int_{0}^{1} \theta(x)\left[u_{t}(t)-p \int_{0}^{x} u_{t}(t-\tau, y) d y\right] \\
& \times\left[u_{x}(t)-\int_{0}^{t} g(t-s) u_{x}(s) d s\right] d x \\
\phi_{2}(t): & \int_{0}^{1}\left[u_{t}(t)-p \int_{0}^{x} u_{t}(t-\tau, y) d y\right] u d x \\
\phi_{3}(t): & =-\int_{0}^{1}\left[u_{t}(t)-p \int_{0}^{x} u_{t}(t-\tau, y) d y\right]\left(\int_{0}^{t} g(t-s)(u(t)-u(s)) d s\right) d x,
\end{aligned}
$$

and

$$
\phi_{4}(t):=e^{-\beta t} \int_{0}^{1} \int_{t-\tau}^{t} e^{\beta(s+\tau)}\left|u_{t}\right|^{2}(s) d s d x, t \geq 0
$$

for some $\beta>0$ (to be determined), where $\|$.$\| stands for the L^{2}$-norm,

$$
\left(g \square u_{x}\right)(t):=\int_{0}^{t} g(t-s)\left|u_{x}(t)-u_{x}(s)\right|^{2} d s,
$$

and $\theta(x)$ is a continuously differentiable function satisfying $\theta(0)=0$ and $\theta(1)=2$ (we may take simply $\theta(x)=2 x$ ).

Observe that the functional $\mathcal{E}(t)$, we shall work with is different form the 'classical' energy

$$
E(t):=\frac{1}{2}\left[\left\|u_{t}\right\|^{2}+\left\|u_{x}\right\|^{2}\right], t \geq 0
$$

This latter functional (2.6) is not convenient to handle because of the appearance of higher order terms in its derivative which are difficult to estimate.

The second functional $\phi_{1}(t)$ is useful to eliminate some undesirable boundary terms which arise in the derivative of $\varepsilon(t)$. The functionals $\phi_{2}(t)$ and $\phi_{3}(t)$ will provide us with $-\left\|u_{x}\right\|^{2}$ and $-\left\|u_{t}\right\|^{2}$, respectively, when differentiated. As for $\phi_{4}(t)$, it will be used to take care of the neutral delay term. Assume that $g$ is a nonnegative nonincreasing function such that

$$
-\xi_{1} g(t) \leq g^{\prime}(t) \leq-\xi_{2} g(t), t \geq 0
$$

for some positive constants $\xi_{1}$ and $\xi_{2}$,

$$
\bar{g}:=\int_{0}^{\infty} g(s) d s<1
$$


and let, for $t_{*}>0$

$$
g_{*}:=\int_{0}^{t_{*}} g(s) d s>0 .
$$

These conditions may be relaxed considerably. One may consider the larger classes of relaxation functions in [13,20-22,23,27-31].

2.1. Lemma. The derivative of $\phi_{1}(t)$, along solutions of (1.1), is estimated as follows:

$$
\begin{aligned}
& \phi_{1}^{\prime}(t) \leq-\left[u_{x}(1)-\int_{0}^{t} g(t-s) u_{x}(s, 1) d s\right]^{2}+\left[\bar{\theta}^{\prime}+\frac{\bar{\theta}}{2}(1+p) g(t)\right]\left\|u_{x}\right\|^{2} \\
& +\left[\left(p+\frac{1}{2}\right) \bar{\theta}^{\prime}+\bar{\theta}\left(p+\delta+\frac{g(t)}{2}\right)\right]\left\|u_{t}\right\|^{2}+\frac{p}{4}\left[\bar{\theta}^{\prime}+\bar{\theta}(1+2 g(t)+4 \delta)\right] \\
& \times\left\|u_{t}(t-\tau)\right\|^{2}+\left[\bar{\theta}^{\prime} \int_{0}^{t} g(s) d s+\frac{\bar{\theta}}{4 \delta} g(0) \xi_{1}(1+p)\right] \int_{0}^{1}\left(g \square u_{x}\right) d x, t \geq 0
\end{aligned}
$$

for some $\delta>0$ and where $\bar{\theta}=\max _{0 \leq x \leq 1} \theta(x)$.

Proof. Clearly, for $t \geq 0$

$$
\begin{aligned}
& \phi_{1}^{\prime}(t)=-\int_{0}^{1} \theta(x)\left(u_{x x}-\int_{0}^{t} g(t-s) u_{x x}(s) d s\right)\left(u_{x}-\int_{0}^{t} g(t-s) u_{x}(s) d s\right) d x \\
& -\int_{0}^{1} \theta(x)\left(u_{t}-p \int_{0}^{x} u_{t}(t-\tau, y) d y\right)\left(u_{x t}-g(0) u_{x}-\int_{0}^{t} g^{\prime}(t-s) u_{x}(s) d s\right) d x
\end{aligned}
$$

To facilitate the estimation later, we write

$$
-g(0) u_{x}-\int_{0}^{t} g^{\prime}(t-s) u_{x}(s) d s=-g(t) u_{x}-\int_{0}^{t} g^{\prime}(t-s)\left[u_{x}(s)-u_{x}(t)\right] d s, t \geq 0 .
$$

Therefore, for $t \geq 0$, we have

$$
\begin{aligned}
& \phi_{1}^{\prime}(t)=-\frac{1}{2} \int_{0}^{1} \theta(x) \frac{\partial}{\partial x}\left(u_{x}-\int_{0}^{t} g(t-s) u_{x}(s) d s\right)^{2} d x-\frac{1}{2} \int_{0}^{1} \theta(x) \frac{\partial\left|u_{t}\right|^{2}}{\partial x} d x \\
& \quad+g(t) \int_{0}^{1} \theta(x) u_{t} u_{x} d x+\int_{0}^{1} \theta(x) u_{t} \int_{0}^{t} g^{\prime}(t-s)\left[u_{x}(s)-u_{x}(t)\right] d s d x \\
& +p \int_{0}^{1} \theta(x) u_{x t} \int_{0}^{x} u_{t}(t-\tau, y) d y d x-p g(t) \int_{0}^{1} \theta(x) u_{x} \int_{0}^{x} u_{t}(t-\tau, y) d y d x \\
& \quad-p \int_{0}^{1} \theta(x)\left(\int_{0}^{x} u_{t}(t-\tau, y) d y\right)\left(\int_{0}^{t} g^{\prime}(t-s)\left[u_{x}(s)-u_{x}(t)\right] d s\right) d x .
\end{aligned}
$$

Applying integration by parts and Young inequality, we get

$$
\begin{gathered}
\phi_{1}^{\prime}(t) \leq-\frac{1}{2}\left[\theta(x)\left(u_{x}-\int_{0}^{t} g(t-s) u_{x}(s) d s\right)^{2}\right]_{0}^{1}-\frac{1}{2}\left[\theta(x)\left|u_{t}\right|^{2}\right]_{0}^{1} \\
+\frac{1}{2} \int_{0}^{1} \theta^{\prime}(x)\left\{\left(1-\int_{0}^{t} g(s) d s\right) u_{x}-\int_{0}^{t} g(t-s)\left[u_{x}(s)-u_{x}(t)\right] d s\right\}^{2} d x \\
+\frac{1}{2} \int_{0}^{1} \theta^{\prime}(x)\left|u_{t}\right|^{2} d x+g(t) \frac{\bar{\theta}}{2}\left\|u_{t}\right\|^{2}+g(t) \frac{\bar{\theta}}{2}\left\|u_{x}\right\|^{2}+\bar{\theta} \delta\left\|u_{t}\right\|^{2} \\
+\frac{\bar{\theta}}{4 \delta} \int_{0}^{1}\left(\int_{0}^{t} g^{\prime}(t-s)\left[u_{x}(s)-u_{x}(t)\right] d s\right)^{2} d x+p\left[\theta(x) u_{t} \int_{0}^{x} u_{t}(t-\tau, y) d y\right]_{0}^{1} \\
-p \int_{0}^{1} \theta^{\prime}(x) u_{t} \int_{0}^{x} u_{t}(t-\tau, y) d y d x-p \int_{0}^{1} \theta(x) u_{t}(t-\tau) u_{t} d x \\
-p g(t) \int_{0}^{1} \theta(x) u_{x} \int_{0}^{x} u_{t}(t-\tau, y) d y d x \\
-p \int_{0}^{1} \theta(x)\left(\int_{0}^{x} u_{t}(t-\tau, y) d y\right)\left(\int_{0}^{t} g^{\prime}(t-s)\left[u_{x}(s)-u_{x}(t)\right] d s\right) d x, t \geq 0 .
\end{gathered}
$$

Further estimations lead to

$$
\begin{gathered}
\phi_{1}^{\prime}(t) \leq \frac{\bar{\theta}^{\prime}}{2}\left\|\left(1-\int_{0}^{t} g(s) d s\right) u_{x}-\int_{0}^{t} g(t-s)\left[u_{x}(s)-u_{x}(t)\right] d s\right\|^{2} \\
-\left[u_{x}(1)-\int_{0}^{t} g(t-s) u_{x}(s, 1) d s\right]^{2}+\left(\frac{\bar{\theta}^{\prime}}{2}+g(t) \frac{\bar{\theta}}{2}+\bar{\theta} \delta\right)\left\|u_{t}\right\|^{2} \\
+\frac{g(t) \bar{\theta}}{2}\left\|u_{x}\right\|^{2}+\frac{\bar{\theta}}{4 \delta}\left(\int_{0}^{t}-g^{\prime}(s) d s\right)\left(\int_{0}^{t}-g^{\prime}(t-s)\left\|u_{x}(t)-u_{x}(s)\right\|^{2} d s\right) \\
+p \bar{\theta}^{\prime}\left\|u_{t}\right\|^{2}+\frac{p \bar{\theta}^{\prime}}{4}\left\|u_{t}(t-\tau)\right\|^{2}+p \bar{\theta}\left\|u_{t}\right\|^{2}+\frac{p \bar{\theta}}{4}\left\|u_{t}(t-\tau)\right\|^{2} \\
\quad+g(t) \frac{p \bar{\theta}}{2}\left[\left\|u_{x}\right\|^{2}+\left\|u_{t}(t-\tau)\right\|^{2}\right]+p \bar{\theta} \delta\left\|u_{t}(t-\tau)\right\|^{2} \\
+\frac{p \bar{\theta}}{4 \delta}\left(\int_{0}^{t}-g^{\prime}(s) d s\right)\left(\int_{0}^{t}-g^{\prime}(t-s)\left\|u_{x}(t)-u_{x}(s)\right\|^{2} d s\right), t \geq 0
\end{gathered}
$$


or

$$
\begin{aligned}
& \phi_{1}^{\prime}(t) \leq-\left[u_{x}(1)-\int_{0}^{t} g(t-s) u_{x}(s, 1) d s\right]^{2}+\left[\bar{\theta}^{\prime}+(1+p) \frac{\bar{\theta}}{2} g(t)\right]\left\|u_{x}\right\|^{2} \\
& \quad+\left[\left(\frac{1}{2}+p\right) \bar{\theta}^{\prime}+g(t) \frac{\bar{\theta}}{2}+(p+\delta) \bar{\theta}\right]\left\|u_{t}\right\|^{2}+\frac{p}{4}\left[\bar{\theta}^{\prime}+\bar{\theta}(1+2 g(t)+4 \delta)\right] \\
& \quad \times\left\|u_{t}(t-\tau)\right\|^{2}+\left[\bar{\theta}^{\prime}\left(\int_{0}^{t} g(s) d s\right)+\frac{(p+1) \bar{\theta}}{4 \delta} g(0) \xi_{1}\right] \int_{0}^{1}\left(g \square u_{x}\right) d x, t \geq 0 .
\end{aligned}
$$

2.2. Lemma. The derivative of $\phi_{2}(t)$, along solutions of (1.1), satisfies

$$
\begin{aligned}
\phi_{2}^{\prime}(t) \leq & \left(1+\delta_{1} p\right)\left\|u_{t}\right\|^{2}+\frac{p}{4 \delta_{1}}\left\|u_{t}(t-\tau)\right\|^{2}-(1-\bar{g}-\delta)\left\|u_{x}\right\|^{2} \\
& +\frac{1}{4 \delta}\left(\int_{0}^{t} g(s) d s\right) \int_{0}^{1}\left(g \square u_{x}\right) d x, t \geq 0
\end{aligned}
$$

for some $\delta, \delta_{1}>0$.

Proof. It is easy to see that, from (2.3) and (1.1), we have

$$
\begin{gathered}
\phi_{2}^{\prime}(t)=\int_{0}^{1} u_{t}\left[u_{t}-p \int_{0}^{x} u_{t}(t-\tau, y) d y\right] d x+\int_{0}^{1} u\left[u_{t t}-\int_{0}^{x} u_{t t}(t-\tau, y) d y\right] d x \\
=\left\|u_{t}\right\|^{2}-p \int_{0}^{1} u_{t} \int_{0}^{x} u_{t}(t-\tau, y) d y d x+\int_{0}^{1} u\left[u_{x x}-\int_{0}^{t} g(t-s) u_{x x}(s) d s\right] d x \\
\leq\left(1+\delta_{1} p\right)\left\|u_{t}\right\|^{2}+\frac{p}{4 \delta_{1}}\left\|u_{t}(t-\tau)\right\|^{2}-\left(1-\int_{0}^{t} g(s) d s\right)\left\|u_{x}\right\|^{2} \\
\quad+\int_{0}^{1} u_{x} \int_{0}^{t} g(t-s)\left[u_{x}(s)-u_{x}(t)\right] d s d x \\
\leq\left(1+\delta_{1} p\right)\left\|u_{t}\right\|^{2}+\frac{p}{4 \delta_{1}}\left\|u_{t}(t-\tau)\right\|^{2}-(1-\bar{g})\left\|u_{x}\right\|^{2} \\
+\delta\left\|u_{x}\right\|^{2}+\frac{1}{4 \delta}\left(\int_{0}^{t} g(s) d s\right) \int_{0}^{1}\left(g \square u_{x}\right) d x
\end{gathered}
$$

for $t \geq 0$ and some $\delta, \delta_{1}>0$. The proof is complete.

2.3. Lemma. The rate of change of the functional $\phi_{3}(t)$, fulfills the estimate

$$
\begin{gathered}
\phi_{3}^{\prime}(t) \leq \delta\left(1-g_{*}\right)^{2} \bar{g}\left\|u_{x}\right\|^{2}+\left(\delta+p \delta_{2} \bar{g}^{2}-g_{*}\right)\left\|u_{t}\right\|^{2} \\
+p\left(\delta+\frac{1}{4 \delta_{2}}\right)\left\|u_{t}(t-\tau)\right\|^{2}+\frac{1}{2}\left(2 \bar{g}+\frac{1+2 g(0) C_{p} \xi_{1}}{2 \delta}\right) \int_{0}^{1}\left(g \square u_{x}\right) d x, t \geq t_{*}
\end{gathered}
$$

for some positive constants $\delta$ and $\delta_{2}$.

Proof. From the definition of $\phi_{3}(t)$, it appears that

$$
\begin{gathered}
\phi_{3}^{\prime}(t)=-\int_{0}^{1}\left(u_{x x}-\int_{0}^{t} g(t-s) u_{x x}(s) d s\right)\left(\int_{0}^{t} g(t-s)(u(t)-u(s)) d s\right) d x \\
-\int_{0}^{1}\left(u_{t}-p \int_{0}^{x} u_{t}(t-\tau, y) d y\right)\left[\left(\int_{0}^{t} g(s) d s\right) u_{t}+\int_{0}^{t} g^{\prime}(t-s)(u(t)-u(s)) d s\right] d x \\
\leq \int_{0}^{1}\left(u_{x}-\int_{0}^{t} g(t-s) u_{x}(s) d s\right)\left(\int_{0}^{t} g(t-s)\left(u_{x}(t)-u_{x}(s)\right) d s\right) d x \\
-\left(\int_{0}^{t} g(s) d s\right)\left\|u_{t}\right\|^{2}+\delta\left\|u_{t}\right\|^{2}+\frac{g(0)}{4 \delta} C_{p} \int_{0}^{1}\left(-g^{\prime} \square u_{x}\right) d x+\delta p\left\|u_{t}(t-\tau)\right\|^{2} \\
+\frac{g(0)}{4 \delta} C_{p} \int_{0}^{1}\left(-g^{\prime} \square u_{x}\right) d x+\left(\int_{0}^{t} g(s) d s\right)^{2} p \delta_{2}\left\|u_{t}\right\|^{2}+\frac{p}{4 \delta_{2}}\left\|u_{t}(t-\tau)\right\|^{2}, t \geq 0
\end{gathered}
$$

where $C_{p}$ is the Poincaré constant, for some positive constants $\delta$ and $\delta_{2}$. Therefore,

$$
\begin{gathered}
\phi_{3}^{\prime}(t) \leq \delta\left(1-\int_{0}^{t} g(s) d s\right)^{2} \bar{g}\left\|u_{x}\right\|^{2}+\left(\frac{1}{4 \delta}+\bar{g}\right) \int_{0}^{1}\left(g \square u_{x}\right) d x+\left(\delta+p \delta_{2} \bar{g}^{2}-g_{*}\right) \\
\quad \times\left\|u_{t}\right\|^{2}+p\left(\delta+\frac{1}{4 \delta_{2}}\right)\left\|u_{t}(t-\tau)\right\|^{2} \quad+\frac{g(0)}{2 \delta} C_{p} \xi_{1} \int_{0}^{1}\left(g \square u_{x}\right) d x, t \geq t_{*} .
\end{gathered}
$$

This finishes the proof.

2.4. Lemma. The derivative of $\phi_{4}(t)$ is equal to

$$
\phi_{4}^{\prime}(t)=-\beta \phi_{4}(t)+e^{\beta \tau}\left\|u_{t}\right\|^{2}-\left\|u_{t}(t-\tau)\right\|^{2}, t \geq 0 .
$$

Proof. Straightforward. 
2.5. Theorem. Let $u_{0}(0) \in H^{1}(0,1)$ and $u_{1} \in L^{\infty}\left([-\tau, 0] ; L^{2}(0,1)\right)$. There exists a $\bar{p}<1$ (depending on $\xi_{2}$ ) such that if $0<p \leq \bar{p}$, then solutions of (1.1) decay to zero in an exponential manner. That is, there exist two positive constants $M$ and $c$ such that

$$
E(t):=\frac{1}{2}\left(\left\|u_{t}\right\|^{2}+\left\|u_{x}\right\|^{2}\right) \leq M e^{-c t}, t \geq 0 .
$$

Proof. First, let us evaluate the derivative of $\mathcal{E}(t)$. Multiplying the equation in (1.1) by the expression

$$
u_{t}(t)-p \int_{0}^{x} u_{t}(t-\tau, y) d y
$$

we find, for $t \geq 0$

$$
\begin{gathered}
\frac{1}{2} \frac{d}{d t} \int_{0}^{1}\left[u_{t}(t)-p \int_{0}^{x} u_{t}(t-\tau, y) d y\right]^{2} d x+\frac{1}{2} \frac{d}{d t}\left\|u_{x}\right\|^{2} \\
=-\int_{0}^{1} u_{t} \int_{0}^{t} g(t-s) u_{x x}(s) d s d x-p \int_{0}^{1} u_{x x} \int_{0}^{x} u_{t}(t-\tau, y) d y d x \\
\quad+p \int_{0}^{1}\left(\int_{0}^{x} u_{t}(t-\tau, y) d y\right)\left(\int_{0}^{t} g(t-s) u_{x x}(s) d s\right) d x \\
=\int_{0}^{1} u_{t x} \int_{0}^{t} g(t-s) u_{x}(s) d s d x+p \int_{0}^{1} u_{t}(t-\tau) u_{x} d x-p u_{x}(1) \int_{0}^{1} u_{t}(t-\tau, y) d y \\
-p \int_{0}^{1} u_{t}(t-\tau) \int_{0}^{t} g(t-s) u_{x}(s) d s d x+p\left(\int_{0}^{1} u_{t}(t-\tau, y) d y\right) \int_{0}^{t} g(t-s) u_{x}(s, 1) d s .
\end{gathered}
$$

The first term in the right hand side of (2.9) is equal to

$$
\begin{gathered}
\int_{0}^{1} u_{t x} \int_{0}^{t} g(t-s) u_{x}(s) d s d x=\frac{1}{2} \int_{0}^{1}\left(g^{\prime} \square u_{x}\right) d x-\frac{1}{2} g(t)\left\|u_{x}\right\|^{2} \\
-\frac{1}{2} \frac{d}{d t}\left\{\int_{0}^{1}\left(g \square u_{x}\right) d x-\left(\int_{0}^{t} g(s) d s\right)\left\|u_{x}\right\|^{2}\right\}, t \geq 0 .
\end{gathered}
$$

Therefore,

$$
\begin{gathered}
\mathcal{E}^{\prime}(t) \leq \frac{1}{2} \int_{0}^{1}\left(g^{\prime} \square u_{x}\right) d x-\frac{1}{2} g(t)\left\|u_{x}\right\|^{2}+p \delta_{3}\left\|u_{x}\right\|^{2} \\
+p \delta_{3}\left(\int_{0}^{t} g(s) d s\right) \int_{0}^{1}\left(g \square u_{x}\right) d x+\frac{p}{2 \delta_{3}}\left\|u_{t}(t-\tau)\right\|^{2} \\
+\frac{p}{2}\left\|u_{t}(t-\tau)\right\|^{2}+\frac{p}{2}\left[u_{x}(1)-\int_{0}^{t} g(t-s) u_{x}(s, 1) d s\right]^{2}, t \geq 0
\end{gathered}
$$

for some $\delta_{3}>0$, or better

$$
\begin{gathered}
\mathcal{E}^{\prime}(t) \leq-\left(\frac{\xi_{2}}{2}-p \delta_{3} \bar{g}\right) \int_{0}^{1}\left(g \square u_{x}\right) d x-\left(\frac{1}{2} g(t)-p \delta_{3}\right)\left\|u_{x}\right\|^{2} \\
+\frac{p}{2}\left(1+\frac{1}{\delta_{3}}\right)\left\|u_{t}(t-\tau)\right\|^{2}+\frac{p}{2}\left[u_{x}(1)-\int_{0}^{t} g(t-s) u_{x}(s, 1) d s\right]^{2}, t \geq 0 .
\end{gathered}
$$

Now, we consider the functional

$$
L(t):=\mathcal{E}(t)+\sum_{i=1}^{4} \lambda_{i} \phi_{i}(t), t \geq 0, \lambda_{i} \geq 0, i=1,2,3,4 .
$$

In view of (2.10) and Lemma 2.1 to Lemma 2.4, we get

$$
\begin{gathered}
L^{\prime}(t) \leq-\left(\frac{\xi_{2}}{2}-p \delta_{3} \bar{g}\right) \int_{0}^{1}\left(g \square u_{x}\right) d x-\left(\frac{1}{2} g(t)-p \delta_{3}\right)\left\|u_{x}\right\|^{2}+\frac{p}{2}\left(1+\frac{1}{\delta_{3}}\right) \\
\times\left\|u_{t}(t-\tau)\right\|^{2}+\frac{p}{2}\left[u_{x}(1)-\int_{0}^{t} g(t-s) u_{x}(s, 1) d s\right]^{2}+\lambda_{1}\left[\bar{\theta}^{\prime}+\frac{\bar{\theta}}{2}(1+p) g(t)\right] \\
\times\left\|u_{x}\right\|^{2}-\lambda_{1}\left[u_{x}(1)-\int_{0}^{t} g(t-s) u_{x}(s, 1) d s\right]^{2}+\lambda_{1}\left[\left(p+\frac{1}{2}\right) \bar{\theta}^{\prime}+\bar{\theta}\left(p+\delta+\frac{g(t)}{2}\right)\right] \\
\times\left\|u_{t}\right\|^{2}+\lambda_{1} \frac{p}{4}\left[\bar{\theta}^{\prime}+\bar{\theta}(1+2 g(t)+4 \delta)\right]\left\|u_{t}(t-\tau)\right\|^{2}+\lambda_{1}\left[\bar{\theta}^{\prime} \bar{g}+\frac{\bar{\theta}}{4 \delta} g(0) \xi_{1}(1+p)\right] \\
\int_{0}^{1}\left(g \square u_{x}\right) d x+\lambda_{2}\left(1+p \delta_{1}\right)\left\|u_{t}\right\|^{2}+\frac{\lambda_{2} p}{4 \delta_{1}}\left\|u_{t}(t-\tau)\right\|^{2}-\lambda_{2}(1-\bar{g}-\delta)\left\|u_{x}\right\|^{2} \\
+\frac{\lambda_{2}}{4 \delta} \bar{g} \int_{0}^{1}\left(g \square u_{x}\right) d x+\lambda_{3} \delta\left(1-g_{*}\right)^{2} \bar{g}\left\|u_{x}\right\|^{2}+\lambda_{3}\left(\delta+p \delta_{2} \bar{g}^{2}-g_{*}\right)\left\|u_{t}\right\|^{2} \\
+\lambda_{3} p\left(\delta+\frac{1}{4 \delta_{2}}\right)\left\|u_{t}(t-\tau)\right\|^{2}+\frac{\lambda_{3}}{2}\left(2 \bar{g}+\frac{1+2 g(0) C_{p} \xi_{1}}{2 \delta}\right) \int_{0}^{1}\left(g \square u_{x}\right) d x \\
-\beta \lambda_{4} \phi_{4}(t)+\lambda_{4} e^{\beta \tau}\left\|u_{t}\right\|^{2}-\lambda_{4}\left\|u_{t}(t-\tau)\right\|^{2},
\end{gathered}
$$


for $t \geq t_{*}$. Arranging different terms there, we find

$$
\begin{gathered}
L^{\prime}(t) \leq-\left\{\frac{\xi_{2}}{2}-\delta_{3} \bar{g} p-\lambda_{1}\left[\bar{\theta}^{\prime} \bar{g}+\frac{\bar{\theta}}{4 \delta} g(0) \xi_{1}(1+p)\right]-\frac{\lambda_{2}}{4 \delta} \bar{g}-\frac{\lambda_{3}}{2}\right. \\
\left.\times\left(2 \bar{g}+\frac{1+2 g(0) C_{p} \xi_{1}}{2 \delta}\right)\right\} \int_{0}^{1}\left(g \square u_{x}\right) d x-\left\{\frac{1}{2} g(t)-\lambda_{1}\left[\bar{\theta}^{\prime}+\frac{\bar{\theta}}{2}(1+p) g(t)\right]\right. \\
\left.-p \delta_{3}+\lambda_{2}(1-\bar{g}-\delta)-\lambda_{3} \delta\left(1-g_{*}\right)^{2} \bar{g}\right\}\left\|u_{x}\right\|^{2}+\left(\frac{p}{2}-\lambda_{1}\right) \\
\times\left[u_{x}(1)-\int_{0}^{t} g(t-s) u_{x}(s, 1) d s\right]^{2}+\left\{\lambda_{1}\left[\left(p+\frac{1}{2}\right) \bar{\theta}^{\prime}+\bar{\theta}\left(p+\delta+\frac{g(t)}{2}\right)\right]\right. \\
\left.+\lambda_{2}\left(1+\delta_{1} p\right)+\lambda_{4} e^{\beta \tau}+\lambda_{3}\left(\delta+p \delta_{2} \bar{g}^{2}-g_{*}\right)\right\}\left\|u_{t}\right\|^{2}+\left\{\frac{p}{2}\left(1+\frac{1}{\delta_{3}}\right)+\frac{\lambda_{2} p}{4 \delta_{1}}\right. \\
\left.+\lambda_{1} \frac{p}{4}\left[\bar{\theta}^{\prime}+\bar{\theta}(1+2 g(t)+4 \delta)\right]+\lambda_{3} p\left(\delta+\frac{1}{4 \delta_{2}}\right)-\lambda_{4}\right\}\left\|u_{t}(t-\tau)\right\|^{2} \\
-\beta \lambda_{4} \phi_{4}(t), t \geq t_{*} .
\end{gathered}
$$

Our objective is to end up with $L^{\prime}(t) \leq-C L(t), \quad t \geq t_{*}$ for some positive constant $C$. Observe that, if all the coefficients in (2.11) are negative (except the one of the boundary term which may be nonpositive), then

$$
L^{\prime}(t) \leq-C_{1}\left\{\left\|u_{t}\right\|^{2}+\left\|u_{x}\right\|^{2}+\int_{0}^{1}\left(g \square u_{x}\right) d x+\left\|u_{t}(t-\tau)\right\|^{2}+\phi_{4}(t)\right\}, t \geq t_{*},
$$

for some $C_{1}>0$. Moreover, using Young inequality and Poincaré inequality, it is easily seen that the right hand side in (2.12) is smaller than or equal to $-C_{2} L(t)$ for some $C_{2}>0$. That is,

$$
L^{\prime}(t) \leq-C_{2} L(t), t \geq t_{*} .
$$

This implies that

$$
L(t) \leq L\left(t_{*}\right) e^{-C_{2}\left(t-t_{*}\right)} \leq L(0) e^{-C_{2}\left(t-t_{*}\right)}, t \geq t_{*} .
$$

Let us first, forget about the first term in the right hand side of (2.11), ignore $\delta$ and ignore $g(t)$ as we may push $t_{*}$ to the right until we obtain a small enough $g(t)$. We need to have

$$
\left\{\begin{array}{l}
p \delta_{3}+\lambda_{1} \bar{\theta}^{\prime}<\lambda_{2}(1-\bar{g}) \\
\frac{p}{2} \leq \lambda_{1} \\
\lambda_{1}\left[\left(p+\frac{1}{2}\right) \bar{\theta}^{\prime}+\bar{\theta} p\right]+\lambda_{2}\left(1+\delta_{1} p\right)+\lambda_{4} e^{\beta \tau}<\lambda_{3}\left(g_{*}-p \delta_{2} \bar{g}^{2}\right), \\
\frac{p}{2}\left(1+\frac{1}{\delta_{3}}\right)+\lambda_{1} \frac{p}{4}\left(\bar{\theta}^{\prime}+\bar{\theta}\right)+\frac{\lambda_{2} p}{4 \delta_{1}}+\frac{\lambda_{3} p}{4 \delta_{2}}<\lambda_{4} .
\end{array}\right.
$$

To fix ideas, let $\theta(x)=2 x, \delta_{1}=\delta_{3}=1, \lambda_{1}=p / 2, \lambda_{2}=4 p /(1-\bar{g})$. Then, we need

$$
\left(2 p+\frac{1}{2}\right) p+\frac{4 p(1+p)}{1-\bar{g}}+\lambda_{4} e^{\beta \tau}<\lambda_{3}\left(g_{*}-p \delta_{2} \bar{g}^{2}\right)
$$

and

$$
4 p+p^{2}+\frac{4 p^{2}}{1-\bar{g}}+\frac{\lambda_{3} p}{\delta_{2}}<4 \lambda_{4}
$$

Combining these two inequalities, we see that it is possible to select $\lambda_{3}$ and $\lambda_{4}$ provided that

$$
g_{*}-p \delta_{2} \bar{g}^{2}-\frac{p}{4 \delta_{2}}>0
$$

that is

$$
4 p \bar{g}^{2} \delta_{2}^{2}-4 g_{*} \delta_{2}+p<0 .
$$


This requires $p<g_{*} / \bar{g}$ and $\delta_{2}$ between the two positive roots of

$$
4 p \bar{g}^{2} x^{2}-4 g_{*} x+p=0 .
$$

Notice that as $t_{*}$ is large then $g_{*} / \bar{g}$ is close to 1 .

Note that $e^{\beta \tau}$ may be ignored as it can be made very close to 1 by considering $\beta$ small enough. Therefore, all the inequalities are satisfied for $p$ small, namely when

$$
\left\{\begin{array}{l}
p\left(1+\bar{\theta}^{\prime}\right)<\lambda_{2}(1-\bar{g}), \\
p\left[\left(p+\frac{1}{2}\right) \bar{\theta}^{\prime}+\bar{\theta} p\right]<\frac{\lambda_{3}}{6} g_{*}, \\
p\left[1+\frac{p}{4}\left(\bar{\theta}^{\prime}+\bar{\theta}\right)\right]<\frac{\lambda_{4}}{3} .
\end{array}\right.
$$

Next, we select $\delta$ small enough and $t_{*}$ large enough so that all the coefficients in $(2.11)$ are negative (except the first one in the right side of (2.11)).

The first term in the right hand side of (2.11) is negative if $A p<\xi_{2} / 2$ where $A$ is a positive constant which is known after our previous selections. This requires a restriction on $p$ in terms of $\xi_{2}$. See the remark after the proof regarding the range of $p$.

Clearly

$$
\left\|u_{t}(t)-p \int_{0}^{x} u_{t}(t-\tau, y) d y\right\|^{2} \leq 2 L(t), t \geq t_{*} .
$$

Therefore,

$$
\begin{gathered}
\left\|u_{t}\right\| \leq\left\|u_{t}(t)-p \int_{0}^{x} u_{t}(t-\tau, y) d y\right\|+p\left\|u_{t}(t-\tau)\right\| \\
\leq \sqrt{2 L(0)} e^{-C_{2}\left(t-t_{*}\right) / 2}+p\left\|u_{t}(t-\tau)\right\| \\
\leq C_{3} e^{-C_{2}\left(t-t_{*}\right) / 2}+p\left\|u_{t}(t-\tau)\right\|, C_{3}=\sqrt{2 L(0)}, t \geq t_{*}
\end{gathered}
$$

This leads to

$$
\left\|u_{t}\right\| \leq C_{4} e^{-a\left(t-t_{*}\right)}+e^{-b\left(t-t_{*}\right)} \sup _{t_{*}-\tau \leq \sigma \leq t_{*}}\left\|u_{t}(\sigma)\right\|, t \geq t_{*}
$$

for some $a, b>0$ and $C_{4}>C_{3}$ (to be determined). Indeed, notice first, that (2.14) holds at $t_{*}$. Assume that there exists $\bar{t}>t_{*}$ such that

$$
\left\|u_{t}(\bar{t})\right\|=C_{4} e^{-a\left(\bar{t}-t_{*}\right)}+e^{-b\left(\bar{t}-t_{*}\right)} \sup _{t_{*}-\tau \leq \sigma \leq t_{*}}\left\|u_{t}(\sigma)\right\|
$$

and

$$
\left\|u_{t}(t)\right\|<C_{4} e^{-a\left(t-t_{*}\right)}+e^{-b\left(t-t_{*}\right)} \sup _{t_{*}-\tau \leq \sigma \leq t_{*}}\left\|u_{t}(\sigma)\right\|, t \in\left[t_{*}, \bar{t}\right) .
$$

Suppose that $\bar{t}-\tau \in\left[t_{*}, \bar{t}\right)$ i.e. $\bar{t} \geq t_{*}+\tau$, then

$$
\begin{gathered}
\left\|u_{t}(\bar{t})\right\| \leq C_{3} e^{-C_{2}\left(\bar{t}-t_{*}\right) / 2}+p\left\|u_{t}(\bar{t}-\tau)\right\| \\
<C_{3} e^{-C_{2}\left(\bar{t}-t_{*}\right) / 2}+p\left[C_{4} e^{-a\left(\bar{t}-\tau-t_{*}\right)}+e^{-b\left(\bar{t}-\tau-t_{*}\right)} \sup _{t_{*}-\tau \leq \sigma \leq t_{*}}\left\|u_{t}(\sigma)\right\|\right] \\
=\left(C_{3}+p C_{4} e^{a \tau}\right) e^{-a\left(\bar{t}-t_{*}\right)}+p e^{b \tau} e^{-b\left(\bar{t}-t_{*}\right)} \sup _{t_{*}-\tau \leq \sigma \leq t_{*}}\left\|u_{t}(\sigma)\right\|
\end{gathered}
$$

provided that $a \leq C_{2} / 2$. To reach a contradiction with (2.15), we need to have

$$
C_{3}+p C_{4} e^{a \tau} \leq C_{4} \text { and } p e^{b \tau} \leq 1
$$

As $p<1$, we can always pick $C_{4}=\frac{C_{3}}{1-p e^{a \tau}}$ with $p e^{a \tau}<1$ and $p e^{b \tau} \leq 1$. That is, $a$ and $b$ exist. Therefore,

$$
\left\|u_{t}\right\| \leq \frac{C_{3}}{1-p e^{a \tau}} e^{-a\left(t-t_{*}\right)}+e^{-b\left(t-t_{*}\right)} \sup _{t_{*}-\tau \leq \sigma \leq t_{*}}\left\|u_{t}(\sigma)\right\|
$$


for $t \geq t_{*}$ with the parameters as above. If $t_{*}<\bar{t}<t_{*}+\tau$ i.e. $\bar{t}-\tau<t_{*}$, then

$$
\left\|u_{t}(\bar{t}-\tau)\right\| \leq \sup _{t_{*}-\tau \leq \sigma \leq t_{*}}\left\|u_{t}(\sigma)\right\| .
$$

Therefore,

$$
\left\|u_{t}(\bar{t})\right\| \leq C_{3} e^{-C_{2}\left(\bar{t}-t_{*}\right) / 2}+p e^{b\left(\bar{t}-t_{*}\right)} e^{-b\left(\bar{t}-t_{*}\right)}\left\|u_{t}(\bar{t}-\tau)\right\| .
$$

If $p e^{b\left(\bar{t}-t_{*}\right)} \leq 1$ which holds if $p e^{b \tau} \leq 1$, then

$$
\begin{gathered}
\left\|u_{t}(\bar{t})\right\| \leq C_{3} e^{-a\left(\bar{t}-t_{*}\right)}+e^{-b\left(\bar{t}-t_{*}\right)} \sup _{t_{*}-\tau \leq \sigma \leq t_{*}}\left\|u_{t}(\sigma)\right\| \\
<C_{4} e^{-a\left(\bar{t}-t_{*}\right)}+e^{-b\left(\bar{t}-t_{*}\right)} \sup _{t_{*}-\tau \leq \sigma \leq t_{*}}\left\|u_{t}(\sigma)\right\|
\end{gathered}
$$

and we reach again a contradiction. The assertion in the theorem is proved for $t \geq t_{*}$ and it holds also on $\left[0, t_{*}\right]$.

2.6. Remark. For the last part of the proof of the theorem we required $0<p<1$ and before that we have imposed some smallness conditions on $p$. In fact, $p$ may be very close to one. This depends on $\xi_{2}$; if $\xi_{2}$ is large enough then these conditions are minor and not very restrictive. That is, $p$ need not be very small. The reader will notice easily that we did not manage any effort in improving these conditions. It would be interesting to determine an "optimal" or "critical" number $p^{*}$ beyond which we get some kind of instability.

Acknowledgment: The author is grateful for the financial support and the facilities provided by King Fahd University of Petroleum and Minerals through project No. IN151015.

\section{References}

[1] Boltzmann L. Zur theorie der elastischen nachwirkung, Ann. Phys. Chem 7, 624-654, 1876.

[2] Bontsema J. and De Vries S. A. Robustness of flexible systems against small time delays, in Proc. 27th Conference on Decision and Control, Austin, Texas, Dec. 1988.

[3] Christensen C. M., Theory of Viscoelasticity, Academic Press 1971.

[4] Coleman B. D. and Gurtin M. E., Waves in materials with memory II, Arch. Rational Mech. Anal. 19, 239-265, 1965.

[5] Dafermos C. M. Asymptotic stability in viscoelasticity, Arch. Rational Mech. Anal. 37, 297308,1970

[6] Datko R. Not all feedback stabilized hyperbolic systems are robust with respect to small time delays in their feedbacks, SIAM J. Control Optim., 26, 697-713, 1988.

[7] Datko R., Lagnese J. and Polis M. P. An example of the effect of time delays in boundary feedback stabilization of wave equations, SIAM J. Control Optim. 24, 152-156, 1986.

[8] Day, N. A. Thermodynamics of Simple Materials with Fading Memory, Springer-Verlag, Berlin 1972.

[9] Desch W., Hannsgen K. B., Renardy Y. and Wheeler R. L. Boundary stabilization of an Euler-Bernoulli beam with viscoelastic damping, in Proc. 26th Conference on Decision and Control, Los Angeles, CA, Dec. 1987.

[10] Eduardo Hernández M. and Henriquez H. R. Existence results for second order partial neutral functional differential equations, Dyn. Cont. Discrete Impul. Syst. Series A: Math. Anal. 15, 645-670, 2008.

[11] Eduardo Hernández M., Henriquez H. R. and McKibben M. A. Existence of solutions for second order partial neutral functional differential equations, Integr. Equ. Oper. Theory 62, 191-217, 2008.

[12] Grimmer R., Lenczewski R. and Schappacher W. Well-posedness of hyperbolic equations with delay in the boundary conditions, in Semigroup Theory and Applications, P. Clement, S. Invernizzi, E. Mitidieri, and I. Vrabie, eds., Lecture Notes in Pure and Appl. Math. 116 (Marcel Dekker, New York and Basel, 1989). 
[13] Guesmia A. Asymptotic stability of abstract dissipative systems with infinite memory, J. Math. Anal. Appl. 382, 748-760, 2011.

[14] Hale J. K. Theory of Functional Differential Equations, (Springer-Verlag, New York, 1997).

[15] Hale J. K. and Verduyn Lunel S.M. Introduction to Functional Differential Equations, in: Applied Mathematical Sciences, 99 (Springer Verlag, New York, 2003).

[16] Hannsgen K. B., Renardy Y. and Wheeler R. L. Effectiveness and robustness with respect to time delays of boundary feedback stabilization in one-dimensional viscoelasticity, SIAM J. Control Optim. 26 (1988), 1200-1234.

[17] Kolmanovskii V. and Myshkis A. Introduction to the Theory and Applications of Functional Differential Equations, (Kluwer Academic Publishers, Dordrecht, 1999).

[18] Liu G. and Yan J. Global asymptotic stability of nonlinear neutral differential equation, Commun Nonlinear Sci. Numer. Simulat. 19, 1035-1041, 2014.

[19] MacCamy R. C. A model for one-dimensional nonlinear viscoelasticity, Quart. Appl. Math. 35, 21-33, 1977.

[20] Medjden M. and Tatar N.-e. Asymptotic behavior for a viscoelastic problem with not necessarily decreasing kernel, Appl. Math. Comput. 167 (2), 1221-1235, 2005.

[21] Medjden M. and Tatar N.-e. On the wave equation with a temporal nonlocal term, Dynamic Systems and Applications 16, 665-672, 2007.

[22] Messaoudi S. General decay of solutions of a viscoelastic equation, J. Math. Anal. Appl. 341 (2), 1457-1467, 2008.

[23] Pata V. Exponential stability in linear viscoelasticity, Quart. Appl. Math. LXIV (3), 499513, 2006.

[24] Renardy M, Hrusa W. J. and Nohel J. A., Mathematical Problems in Viscoelasticity, Pitman Monographs and Surveys in Pure and Applied Mathematics, 35, Longman Scientific and Technical, Hohn Wiley and Sons, New York, 1987.

[25] Rojsiraphisal T. and Niamsup P. Exponential stability of certain neutral differential equations, Appl. Math. Comput. 217, 3875-3880, 2010.

[26] Sánchez J. and Vergara V. Long-time behavior of nonlinear integro-differential evolution equations, Nonlin. Anal.: T. M. A. 91, 20-31, 2013.

[27] Tatar N.-e. On a problem arising in isothermal viscoelasticity, Int. J. Pure and Appl. Math. 3 (1), 1-12, 2003.

[28] Tatar N.-e. Exponential decay for a viscoelastic problem with singular kernel, Zeit. Angew. Math. Phys. 60 (4), 640-650, 2009.

[29] Tatar N.-e. On a large class of kernels yielding exponential stability in viscoelasticity, Appl. Math. Comp. 215 (6), 2298-2306, 2009.

[30] Tatar N.-e. Arbitrary decays in linear viscoelasticity, Journal Math. Physics 52 (1), 013502, 2011.

[31] Tatar N.-e. A new class of kernels leading to an arbitrary decay in viscoelasticity, Mediterr. J. Math. 10 (1), 213-226, 2013.

[32] Wang J. Existence and stability of solutions for neutral differential equations with delay, International Conference on Multimedia Technology (ICMT), 2011, 2462-2465, 10.1109/ICMT.2011.6002527.

[33] Wang W., Fan Q., Zhang Y. and Li S. Asymptotic stability of solution to nonlinear neutral and Volterra functional differential equations in Banach spaces, Appl. Math. Comput. 237, 217-226, 2014.

[34] Wang W.-S., Li S.-F. and Yang R.-S. Contractivity and exponential stability of solutions to nonlinear neutral functional differential equations in Banach spaces, Acta Math. Appl. Sinica, English Series 28 (2), 289-304, 2012.

[35] Wen L., Wang W. and Yu Y. Dissipativity and asymptotic stability of nonlinear neutral delay integro-differential equations, Nonlin. Anal. T. M. A. 72, 1746-1754, 2010.

[36] Wu J. Theory and Applications of Partial Functional-Differential Equations, in: Applied Mathematical Sciences, 119 (Springer-Verlag, New York, 1996).

[37] Wu J. and Xia H. Self-sustained oscillations in a ring array of coupled lossless transmission lines, J. Diff. Eqs. 124 (1), 247-278, 1996.

[38] Wu J. and Xia H. Rotating waves in neutral partial functional-differential equations, J. Dynam. Diff. Eqs. 11 (2), 209-238, 1999. 
[39] Xie S. Solvability of impulsive partial neutral second-order functional integro-differential equations with infinite delay, Boundary Value Prob. 2013, 203, 2013.

[40] Ye R. and Zhang G. Neutral functional differential equations of second-order with infinite delays, Electron. J. Diff. Eqs. 2010 (36), 1-12, 2010. 